\title{
Electrical challenges of heteroepitaxial 3C-SiC on silicon
}

\author{
Aiswarya Pradeepkumar ${ }^{1, a}$, D Kurt Gaskill, b, \\ Francesca lacopi $i^{1, c}$ \\ ${ }^{1}$ School of Electrical and Data Engineering, University of Technology Sydney, \\ NSW 2007, Australia \\ ${ }^{2}$ US Naval Research Laboratory, Washington, DC 20375, USA \\ aAiswarya.Pradeepkumar@uts.edu.au, bgaskill@nrl.navy.mil, cfrancesca.iacopi@uts.edu.au
}

\begin{abstract}
Keywords: 3C-SiC, electrical conduction, electrical leakage
Abstract. We have investigated the electrical conduction in epitaxial cubic silicon carbide films on low-doped and high-resistive silicon substrates. The electrical properties of the film/substrate system such as the carrier concentration, carrier mobility, and sheet resistance were evaluated by performing Hall measurements in a van der Pauw configuration at room temperature. For the SiC on low-doped $\mathrm{p}$-Si, we found that the charge carriers in the substrate always dominate the electrical conduction indicating an electrical shorting of the film to the substrate and the absence of a $\mathrm{p} / \mathrm{n}$ junction. Meanwhile, for the $\mathrm{SiC}$ films grown on high-resistive silicon, we found an evidence of current leakage through a silicon region right below the $\mathrm{SiC} / \mathrm{Si}$ interface, generated upon $\mathrm{SiC}$ growth. Leakage resistances in the $\mathrm{k} \Omega$ range obtained from TLM structures made of isolated SiC pillars on highresistive silicon confirmed the presence of a conductive region below the $\mathrm{SiC} / \mathrm{Si}$ interface. This work also shows that this electrical leakage can be supressed using a high-resistive silicon as the substrate and etching away the conductive region below the interface.
\end{abstract}

\section{Introduction}

Epitaxial cubic silicon carbide films on silicon have attracted extensive interest for semiconductor device applications such as high-voltage, high-frequency diodes, and heterojunction bipolar transistor [1]. This is because they can offer access to the properties of the $\mathrm{SiC}$ material such as its wide band gap and high thermal conductivity on the more conventional silicon substrates [2]. Rahimi et al. have shown, however, that the substantial tensile strain generated from the lattice and thermal expansion coefficient mismatch between $3 \mathrm{C}-\mathrm{SiC}$ and silicon, may reduce the band gap in the SiC epitaxial films [3]. Nevertheless, the impact of this phenomenon on the electrical and electronic performance of the epitaxial $\mathrm{SiC}$ films on silicon has not been fully elucidated to date; such information is vital to obtain the optimal performance of devices fabricated from these strained heterojunctions.

We have recently shown that the expected $\mathrm{p}$-n junction between a $p$-type silicon substrate and cubic heteroepitaxial silicon carbide (3C-SiC, naturally grown as unintentionally $n$-type) is either nonexisting or very unstable so that severe leakage or shorting of the epitaxial silicon carbide to the underlying silicon substrate is typically found $[4,5]$. In this work, we aim to understand and model the electrical conduction in the $3 \mathrm{C}-\mathrm{SiC}(100) / \mathrm{Si}(100)$ system and the substrate influence on the conduction using commercial NOVASiC samples that were grown at temperatures between 1300 and $1400^{\circ} \mathrm{C}[6]$. Consequently, we compare the epilayers grown on low-doped $p$-type substrate to those grown on highly resistive substrates, which are typically $n$-type.

\section{Experimental}

We focused on unintentionally doped (thus $n$-type) commercial NOVASiC 3C-SiC(100) films with thickness of $500 \mathrm{~nm}$ epitaxially grown at $1300-1400^{\circ} \mathrm{C}$ [6] on $527 \mu \mathrm{m}$ low-doped $p$-type $\mathrm{Si}(100)$ with resistivity ranging from 1 to $10 \Omega \mathrm{cm}$ as well as on high-resistive $\mathrm{Si}(100)$ substrates with resistivity greater than $10 \mathrm{k} \Omega \mathrm{cm}$. For the electrical characterization, we diced the $S i C / S i$ wafers into $1.1 \times 1.1 \mathrm{~cm}^{2}$ coupons, and sputtered $150 \mathrm{~nm}$ thick nickel contacts (no annealing) onto the four corners using a custom made shadow mask, see Fig. 1a and 1b. The electrical properties such as the carrier concentration, carrier mobility, and sheet resistance were estimated at room temperature by 
performing van der Pauw Hall measurements on the $\mathrm{SiC} / \mathrm{Si}$ samples as well as on representative bare Si substrates using Ecopia HMS 5300 Hall Effect Measurement System. SiC/high-resistive Si van der Pauw structures were etched in an inductively coupled plasma (ICP) system using $\mathrm{SF}_{6}$ gas and oxygen to remove the $\mathrm{SiC}$ layer using the $\mathrm{Ni}$ contacts as a hard mask (see Fig. 1c) and the Hall measurements were repeated at room temperature. Additionally, $\mathrm{SiC}$ on high-resistive $\mathrm{Si}$ samples were patterned into transfer length measurement (TLM) structures consisting of $300 \mathrm{~nm}$ thick, 500 $\mu \mathrm{m}$ wide aluminium contacts deposited using e-beam evaporation (no annealing) followed by acid etching of aluminium and reactive-ion etching of SiC, see Fig. 2. Current-voltage measurements were performed at room temperature to measure the leakage resistances using a HP4145B semiconductor parameter analyser. Afterwards, the silicon in-between the $\mathrm{SiC}$ pillars on the SiC/high-resistive $\mathrm{Si}$ van der Pauw and TLM structures were etched using ICP, and all measurements were repeated, see Fig. $4 \mathrm{a}$ and $4 \mathrm{~b}$.

\section{Results}

Table 1. Hall measured transport characteristics at room temperature. Results are the averaged values extracted from three samples for each type.

\begin{tabular}{llllll}
\hline & Bare $\boldsymbol{p}$-Si & 3C-SiC/p-Si & $\begin{array}{l}\text { Bare high- } \\
\text { resistive Si }\end{array}$ & $\begin{array}{l}\text { 3C-SiC/high- } \\
\text { resistive Si }\end{array}$ & $\begin{array}{l}\text { Removed 3C- } \\
\text { SiC/high- } \\
\text { resistive Si }\end{array}$ \\
\hline \hline Carrier type & Holes & Holes & Electrons & Electrons & Electrons \\
Carrier concentration $\left(\mathbf{c m}^{-2}\right)$ & $1( \pm 0.2) \times 10^{14}$ & $1( \pm 0.2) \times 10^{14}$ & $1( \pm 0.2) \times 10^{10}$ & $3( \pm 0.2) \times 10^{11}$ & $4( \pm 0.2) \times 10^{11}$ \\
Mobility $\left(\mathbf{c m}^{2} / \mathbf{V s}\right)$ & $341( \pm 10)$ & $357( \pm 10)$ & $1220( \pm 10)$ & $1677( \pm 10)$ & $1650( \pm 10)$ \\
Sheet resistance $(\mathbf{\Omega} / \square)$ & $173 \pm 10$ & $166 \pm 10$ & $500 \mathrm{k} \pm 3 \mathrm{k}$ & $12 \mathrm{k} \pm 3 \mathrm{k}$ & $12 \mathrm{k} \pm 3 \mathrm{k}$ \\
\hline \hline
\end{tabular}

a)

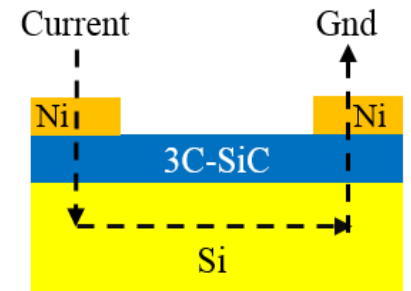

Region involved in conduction b)

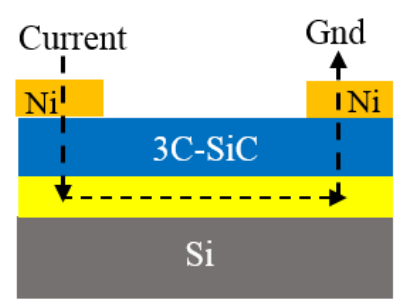

Region involved in conduction c)

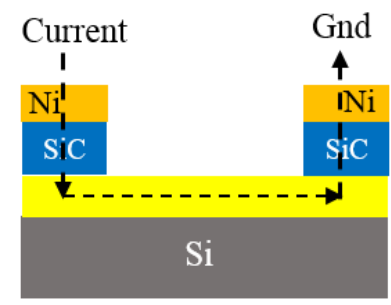

Region involved in conduction

Fig. 1. Schematic of conduction path in the $3 \mathrm{C}-\mathrm{SiC}$ grown at $1300-1400^{\circ} \mathrm{C}$ a) on low-doped $p$-Si substrate; b) on high-resistive $\mathrm{Si}$; c) after etching $\mathrm{SiC}$ layer between the contacts of $\mathrm{SiC} /$ high-resistive $\mathrm{Si}$-the conduction is found to occur within a region of a few micrometres thick below the interface.

From Table 1, the room temperature hall measurement of 3C-SiC grown on a low-doped Si indicates a hole conduction with carrier concentration, mobility and sheet resistance comparable to the bare low-doped $p$-Si substrate. This implies that the $\mathrm{SiC}$ films on low-doped $\mathrm{Si}$ substrates are typically shorted (either upon growth or during subsequent annealing) and the charge carriers in the thick silicon substrate with relatively high mobility dominate the electrical conduction, as shown in Fig. 1a. The electrical shorting persists even if we etch deep into the substrate, as long as the silicon charge carriers exists [5].

The effects of large tensile strain generated from the lattice mismatch as well as the difference in thermal expansion coefficients between the 3C-SiC film and the thick $\mathrm{Si}$ substrate leads to high defect densities [2] as well as changes in the band alignment and band structure at the $3 \mathrm{C}-\mathrm{SiC} / \mathrm{Si}$ heterojunction [3]. The band distortion may make the band gap of $\mathrm{SiC}$ more compatible with silicon. We thus hypothesize that the leakage or the shorting phenomenon of the $3 \mathrm{C}-\mathrm{SiC}$ to the $\mathrm{Si}$ substrate is linked to a combination of increased hole current injection from $\mathrm{Si}$ to $\mathrm{SiC}$ due to a smaller valence band barrier, and the presence of electrically active defects at the $\mathrm{SiC} / \mathrm{Si}$ interface. 
Meanwhile, for the $\mathrm{SiC}$ films grown on high-resistive $\mathrm{Si}$, we believe that, in addition to the $n$-type conduction within the SiC film which is relatively limited (only about $\sim 10^{11} \mathrm{~cm}^{-2}$ sheet carrier concentration), we also find evidence of conduction through a region just below the SiC/Si interface as shown in Fig. 1b and 1c. To corroborate, the leakage resistances are measured for the SiC/highresistive Si using TLM structures in Fig. 2, see Table 2.

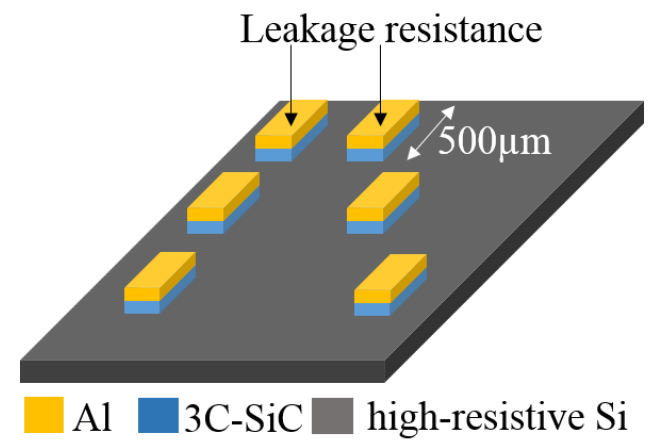

Fig. 2. TLM structures on the 3C-SiC/high-resistive Si for leakage resistance measurement.

Table 2. Leakage resistances across different contact spacing for the SiC TLM structures made on high-resistive $\mathrm{Si}$. Results are the averaged values obtained from two samples.

\begin{tabular}{ll}
\hline \hline $\begin{array}{l}\text { Contact spacing } \\
(\boldsymbol{\mu m})\end{array}$ & $\begin{array}{l}\text { Leakage } \\
\text { resistance } \\
(\mathbf{k} \boldsymbol{\Omega})\end{array}$ \\
\hline \hline 40 & 75 \\
60 & 80 \\
80 & 85 \\
100 & 87 \\
160 & 90 \\
200 & 95 \\
250 & 100 \\
300 & 105 \\
\hline \hline
\end{tabular}

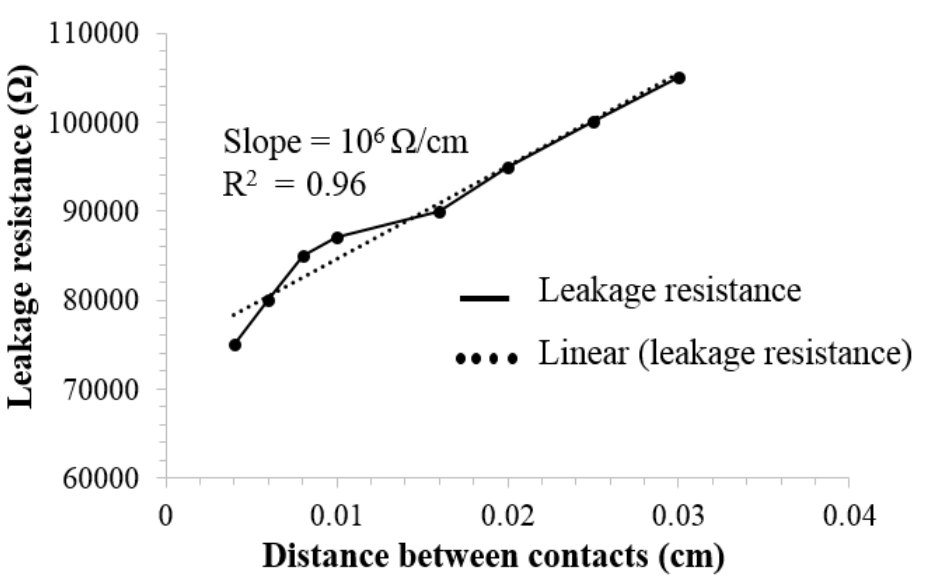

Fig. 3. Fitted TLM leakage resistances versus contact spacing for the $\mathrm{SiC}$ on high-resistive $\mathrm{Si}$.

The data in Table 2 indicate that for $\mathrm{SiC}$ on high-resistive $\mathrm{Si}$, the leakage resistances are larger than that for SiC on low-doped Si [5]. Fig. 3 shows the fitted leakage resistances obtained using TLM structures on the SiC/high-resistive $\mathrm{Si}$ as a function of different contact spacing with a slope of $10^{6}$ $\Omega / \mathrm{cm}$. The sheet resistance calculated from slope and width of the contact is $\sim 50 \mathrm{k} \Omega / \square-$ slightly larger than the sheet resistance of SiC/high-resistive Si obtained using the van der Pauw Hall measurement in Table 1. Note that the actual leakage resistances must be at least one order of magnitude larger than the measured leakage resistance values, for the TLM sheet resistance to agree with the van der Pauw sheet resistance of the bare high-resistive Si substrate $(500 \mathrm{k} \Omega / \square)$, to indicate no leakage.

When using a high-resistive $n$-type silicon substrate, we have a large sheet resistance of $500 \mathrm{k} \Omega / \square$ with limited carriers and thus no p-n junction at the SiC/Si interface. Therefore, any band distortion induced by the interfacial strain in the SiC film may not lead to carrier injection as in the case of lowdoped $p$-Si substrates. Indeed, transport measurements of the $\mathrm{SiC}$ on high-resistive $\mathrm{Si}$ indicate $n$-type majority carriers, which are the carrier type expected in heteroepitaxial 3C-SiC films [4]. However, we also notice in Table 1 that even after total removal of the $\mathrm{SiC}$ layer, those carriers persist. We explain this by suggesting that a highly defective region is formed below the interface upon the $\mathrm{SiC}$ growth, where the carriers of the $\mathrm{SiC}$ film are concentrated.

We can suppress the in-plane electrical leakage of 3C-SiC/high-resistive Si by etching the conductive region for at least $20 \mu \mathrm{m}$ deep as demonstrated in Fig. 4. After etching the silicon in between the $\mathrm{SiC}$ 
pillars for more than $20 \mu \mathrm{m}$ deep until the conductive region in the substrate is eliminated, we isolate the SiC pillars on the van der Pauw and TLM structures (see Table 3 for Hall measurement and TLM leakage resistance results before and after the silicon etching).

a)

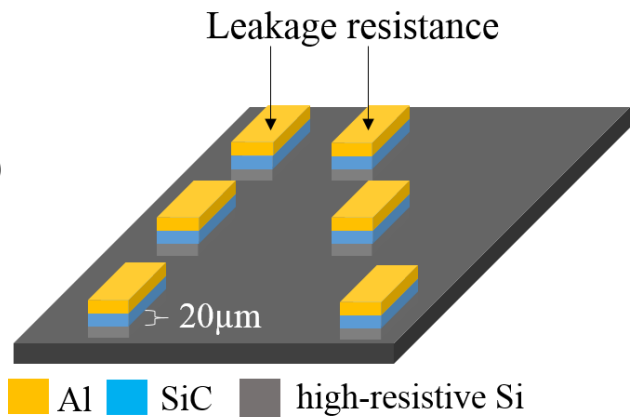

b)

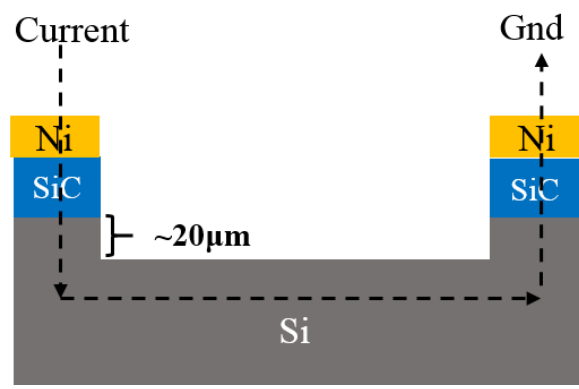

Fig. 4. a) SiC/high-resistive Si TLM structure after $\sim 20 \mu \mathrm{m}$ deep etching of $\mathrm{Si}$; b) electrical conduction in $\mathrm{SiC} /$ high-resistive $\mathrm{Si}$ after $\sim 20 \mu \mathrm{m}$ deep etching of $\mathrm{Si}$

Table 3. Electrical characteristics at room temperature for SiC/high-resistive Si before and after 20 $\mu \mathrm{m}$ deep etching of silicon between SiC pillars a) van der Pauw Hall measurement results b) TLM leakage resistance results. Results after etching are the averaged values of two samples each.

a)

\begin{tabular}{lll}
\hline \hline & $\begin{array}{l}\text { 3C-SiC/high-resistive Si } \\
\text { (before etching) }\end{array}$ & $\begin{array}{l}\text { 3C-SiC/high-resistive Si } \\
\text { (after 20 } \boldsymbol{\mu m} \text { etch) }\end{array}$ \\
\hline \hline Carrier type & Electrons & Electrons \\
Sheet carrier concentration $\left(\mathbf{c m}^{-2}\right)$ & $3( \pm 0.2) \times 10^{11}$ & $5( \pm 0.2) \times 10^{11}$ \\
Mobility $\left(\mathbf{c m}^{2} / \mathrm{Vs}\right)$ & $1677( \pm 10)$ & $34( \pm 10)$ \\
Sheet resistance $(\boldsymbol{\Omega} / \square)$ & $12 \mathrm{k} \pm 3 \mathrm{k}$ & $492 \mathrm{k} \pm 2 \mathrm{k}$ \\
\hline \hline
\end{tabular}

b)

\begin{tabular}{llc}
\hline \hline Contact spacing & \multicolumn{1}{c}{ TLM leakage resistance } \\
\hline \hline$(\boldsymbol{\mu m})$ & before etch $(\mathbf{k} \boldsymbol{\Omega})$ & after $20 \boldsymbol{\mu}$ etch $(\mathbf{M} \boldsymbol{\Omega})$ \\
\hline 40 & 75 & 8.5 \\
60 & 80 & 8.5 \\
80 & 85 & 9.5 \\
100 & 87 & 10 \\
160 & 90 & - \\
200 & 95 & 10 \\
\hline \hline
\end{tabular}

\section{Summary}

Heteroepitaxial 3C-SiC films grown on low-doped $\mathrm{Si}$ are typically electrically shorted to the substrate. This could be due to the presence of a range of electrically active extended defects at the $\mathrm{SiC} / \mathrm{Si}$ interface, together with an increased hole current injection from the $\mathrm{Si}$ to the $\mathrm{SiC}$ generated by the intrinsic strain relaxation in the SiC film. Room temperature van der Pauw and TLM measurement results have shown that this electrical leakage/shorting of the $\mathrm{SiC}$ to the Si substrate can be overcome by using a high-resistive silicon as the substrate and etching away more than $20 \mu \mathrm{m}$ deep into the silicon between the SiC pillars.

\section{References}

[1] S. Nishino et al., J. Electrochem. Soc. 127(12) (1980) 2674-2680.

[2] X. Song et al., Appl. Phys. Lett. 96(14) (2010) 142104-142104-3.

[3] R. Rahimi et al., J. Phys. D: Appl. Phys. 42(5) (2009) 055108.

[4] A. Pradeepkumar et al., Appl. Phys. Lett. 109 (2016) 011604-011604-5.

[5] A. Pradeepkumar et al., Appl. Phys. Lett. 109 (2016) 196102-196102-2.

[6] M. Zielinski et al., Mater. Sci. Eng. B 165(1) (2009) 9-14. 